\title{
Micromechanics of temperature sensitive microgels: dip in the Poisson ratio near the LCST $\uparrow$
}

\author{
Panayiotis Voudouris, ${ }^{\text {ab }}$ Daniel Florea, ${ }^{\text {ab }}$ Paul van $\operatorname{der}_{\text {Schoot }}{ }^{\mathrm{bc}}$ and Hans M. Wyss ${ }^{\star a b}$ \\ Microgels of poly- $N$-isopropylacrylamide (pNIPAM) exhibit a remarkable sensitivity to environmental \\ conditions, most strikingly a pronounced deswelling that occurs close to the lower critical solution \\ temperature (LCST) of the polymer at $\approx 32^{\circ} \mathrm{C}$. This transition has been widely studied and exploited in a \\ range of applications. Along with changes in size, significant changes are also expected for the \\ mechanical response of the particles. However, the full elastic properties of these particles as a function \\ of temperature, $T$, have not yet been assessed at the single-particle level. Here we present \\ measurements of the elastic properties of pNIPAM particles as a function of both temperature and \\ cross-linking density using capillary micromechanics, a technique based on the pressure-dependent \\ deformation of particles trapped in a tapered glass capillary. The shear elastic modulus $G$ increased \\ monotonously upon increasing temperature. In contrast, but in qualitative agreement with previous \\ experiments on macroscopic pNIPAM hydrogels, we found that the compressive elastic modulus $K$ of \\ our microgels exhibits a dip close to the LCST. Remarkably, this dip is less sharp and deep than that \\ observed in macroscopic hydrogels. The Poisson ratio of the particles also exhibits a pronounced dip \\ close to the LCST, reaching unusually low minimum values of $\sigma \approx 0.15$. To rationalize this behavior, we \\ compared our experimental data to Flory-Rehner theory; the theory is able to qualitatively predict the \\ general mechanical behavior observed, thus indicating that the observed dip in the Poisson ratio can be \\ accounted for by simple thermodynamic arguments.
}

\section{Introduction}

Microgels combine the intriguing properties of both polymeric networks and colloidal particles in a single material. ${ }^{1}$ Like polymeric gels they exhibit very pronounced swelling/collapse transitions as a function of physicochemical conditions such as ionic strength, $\mathrm{pH}$, solvent quality, or temperature. ${ }^{2-6}$ Like colloids they are particles with a size ranging from tens of nanometers up to tens of micrometers.

However, as a result of this combination of contrasting properties, their behavior also differs in important ways from those found in both conventional colloids and in bulk polymer gels. Unlike hard colloids, microgel particles can change both their volume as well as their shape due to their polymer-like flexibility. ${ }^{7-11}$ As a result, the concentration dependence of their viscoelastic properties is much less pronounced than that of

${ }^{a}$ Eindhoven University of Technology, Department of Mechanical Engineering, $5612 \mathrm{AZ}$ Eindhoven, The Netherlands. E-mail: H.M.Wyss@tue.nl; Fax: +31 40244 7355; Tel: +31402472920

${ }^{b}$ Eindhoven University of Technology, Institute for Complex Molecular Systems, $5612 A Z$ Eindhoven, The Netherlands

${ }^{\circ}$ Eindhoven University of Technology, Department of Physics, 5612AZ Eindhoven, The Netherlands

$\dagger$ Electronic supplementary information (ESI) available. See DOI: 10.1039/c3sm50917f conventional colloids. For hard particles the transition from a liquid-like to a solid-like behavior occurs within just a few percent in volume fraction, a comparable change in behavior in soft microgels is much more gradual and can require an increase of more than an order of magnitude in concentration. ${ }^{8,12}$ Unlike bulk hydrogels, microgel particles respond much more readily to changes in environmental conditions, with a response to these changes occurring within seconds or minutes rather than hours or even days. ${ }^{13-15}$ This qualitative change in response time is caused by an additional length scale, the particle size, which is not present in bulk hydrogels. As a result, by tuning this length scale as well as the polymer concentration and the cross-linking density, the response of these materials can be sensitively tuned, making them ideally suited for applications such as drug-delivery materials, pharmaceutical systems, or for responsive scaffold materials in tissue-engineering applications. ${ }^{2,16-19}$ Microgels are also often used as additives in personal care products, foods, and in advanced oil recovery, where their tunable material properties are exploited for precisely controlling the macroscopic viscoelastic properties of the material. ${ }^{5,20-23}$

Despite the widespread use of these materials in these practical applications, as well as in applied and fundamental scientific studies, their macroscopic mechanical properties are still poorly understood. Such understanding must link single 
particle properties to properties at the macroscopic scale. Probing mechanical properties at the single particle level is thus of key importance.

For poly- $N$-isopropylacrylamide (pNIPAM) microgels, a dramatic collapse transition occurs as the temperature is increased towards the lower critical solution temperature (LCST) of the polymer. Accompanying this collapse transition, we would expect significant changes in the mechanical properties of these particles. Indeed, recent atomic force microscopy (AFM) measurements on single pNIPAM microgel particles have revealed a pronounced softening of the particles in the vicinity of the LCST. ${ }^{7}$ However, these measurements have been limited to measuring only a single mode of deformation: the Young's modulus $E$ of the particles was extracted by assuming a fixed value of the Poisson ratio $\sigma$. In a similar AFM-based study, the Young's modulus of pNIPAM particles was found to increase continuously with temperature ${ }^{24}$ in this case the authors even assumed an incompressible material $(\sigma=0.5)$ in their analysis of the Young's modulus. The absence of softening in these experiments could also be explained by the fact that the particles used exhibited a significant gradient in the internal crosslinking density, ${ }^{25}$ as is often observed for particles synthesized by emulsion polymerization. ${ }^{26}$ In another study, by applying an external osmotic pressure to pNIPAM particles, the compressive elastic modulus $K$ of single particles was measured directly, revealing a non-monotonic behavior of $K$ as a function of temperature in the vicinity of the LCST. ${ }^{27}$ While these measurements ensure a pure compressive mode of deformation and should thus provide an accurate measure of the compressive modulus, no information on the shear elastic modulus or the Poisson ratio can be obtained.

Ideally, we would like to simultaneously measure both the compressive and the shear modulus of the particles, which together fully characterize the linear elastic behavior of the particles, including the Young's modulus and the Poisson ratio of the material. In this paper we report direct measurements of the dramatic changes in elastic properties of single pNIPAM microgel particles as a function of temperature, crossing the collapse transition that occurs around the LCST of the bulk material. Using the recently developed capillary micromechanics method, ${ }^{\mathbf{1 1 , 2 8 , 2 9}}$ we have measured the compressive elastic modulus $K$ and the shear elastic modulus $G$ over the entire transition region. We observed dramatic changes in both these quantities in the vicinity of the LCST. Most notably we found a pronounced drop of the compressive modulus around the LCST, accompanied by a simultaneous increase of the shear modulus. As a result, the Poisson ratio $\sigma$ of the material drops dramatically, reaching values close to zero, as has been previously observed in macroscopic pNIPAM hydrogels ${ }^{30,31}$ and for other polymer gel materials. ${ }^{32}$

We account for these pronounced changes in material behavior by comparing our experiments to predictions from Flory-Rehner theory, ${ }^{33,34}$ which we find to be in adequate but not perfect agreement with our experimental observations. In particular, the model does not enable us to quantitatively account for the temperature-dependent particle deswelling and simultaneously the change in elastic properties of the particles using the same model parameters. However, qualitatively the theory is able to predict the general behavior observed, thus indicating that the observed dip in the Poisson ratio can be accounted for by simple thermodynamic arguments. We attribute this discrepancy to limitations of the simple theory used, which predicts a much sharper collapse transition than observed in the experiment.

\section{Experimental}

\subsection{Synthesis of microgel particles}

We produced our poly- $N$-isopropylacrylamide (pNIPAM) particles by first making droplets of aqueous monomer solution using microfluidic devices; subsequently the droplets were polymerized to form pNIPAM microgel particles. To fabricate droplets of controlled, uniform size, we used poly-dimethylsiloxane (PDMS) flow focusing microfluidic devices of $60 \mu \mathrm{m}$ channel width, fabricated through soft lithography. ${ }^{35}$

To vary the properties of the particles, we produced four batches of aqueous pre-polymer solution with different concentrations of the cross-linker $\left(N, N_{0}\right.$-methylene-bis-acrylamide: $0.25,0.5,0.7$ and $1.0 \mathrm{wt} \%$ ), while keeping the concentration of the monomer $N$-isopropylacrylamide (NIPAM) fixed at $10 \mathrm{wt} \%(10 \mathrm{mM})$. These concentrations correspond to crosslinker-to-monomer molar ratios of 1/36, 1/14, 1/10, and 1/7, respectively. To eventually induce polymerization, we also added 0.2 wt\% of photo-initiator (2-hydroxy-4'-(2-hydroxyethoxy)-2-methylpropiophenone, Sigma-Aldrich) in the aqueous phase. For the oil phase, we used a poly-dimethylsiloxane (PDMS) fluid ( $\eta=50 \mathrm{cSt}$, Sigma-Aldrich) containing a surfactant (2 wt\%, Abil EM-90, Evonik Industries) to decrease the surface tension of the water-oil interface and to stabilize the forming droplets against coalescence.

The oil and the water phases were infused into the microfluidic device through polyethylene tubing (Scientific Commodities, $0.86 \mathrm{~mm}$ inner diameter) using positive displacement syringe pumps (Pump Nexus 3000). We monitored the droplet formation with a digital camera (Moticam 2000) attached to an inverted optical microscope (Motic AE31) to make sure the device operated in a dripping regime, where droplets of highly uniform size distribution are produced. These droplets were then collected in a Petri dish filled with oil and irradiated with a UV lamp (Vilber-Lourmart, model VL215.LC) for 5 minutes, resulting in photopolymerization of the aqueous droplets and the formation of pNIPAM microgel particles. Particles were subsequently washed by a series of dilution and centrifugation steps, thereby removing the oil phase and the surfactant. After swelling in water at room temperature, the particles exhibited a narrow size distribution with an average diameter larger than the initial drop size, the swelling ratio of initial droplets to swollen particles depending on the cross-linker to monomer ratio.

To quantify the volume phase transition of our particles, we used a temperature-controlled chamber (Linkam Scientific Instruments, model TP-93/LNP), mounted on an optical microscope (Zeiss), and imaged the same group of particles as a function of temperature. As shown in the series of images in 
Fig. 1(B), the particle size decreased significantly as the temperature was increased across the LCST. The particle size at each temperature was extracted from the images, averaging over several particles at each temperature. In Fig. 1(A), we plot the particle diameter as a function of temperature for the four different batches of particles studied, which were synthesized with four different cross-linker-to-monomer ratios. To better compare the relative shrinking of the four batches of particles, the temperature-dependent particle diameter is scaled by $d_{0}$, the diameter at $T=40 \mathrm{~K}$, where the particle size has reached a plateau in a fully collapsed state. The observed swelling ratios clearly depend on the cross-linker-to-monomer ratios of the particles. The particles that were synthesized with the lowest initial concentration of the cross-linker (cross-linker to monomer ratio of $1 / 36$ ) exhibit the most pronounced size change, corresponding to a swelling ratio of $\frac{d_{T=23{ }^{\circ} \mathrm{C}}}{d_{T=40{ }^{\circ} \mathrm{C}}} \approx 2.5$. With increasing cross-linker concentration the swelling ratio continuously decreases to a value of 2 for the batch of particles with a cross-linker-to-monomer ratio of $1 / 7$. This deswelling behavior of our microgel particles is largely in line with existing studies of the collapse transition of macroscopic pNIPAM hydrogels ${ }^{31}$ and microgel particles. ${ }^{2}$

\subsection{Capillary micromechanics}

To access both the compressive elastic modulus and the shear elastic modulus of our particles, we employed the recently developed capillary micromechanics technique. ${ }^{\mathbf{1 1 2 9}}$ The

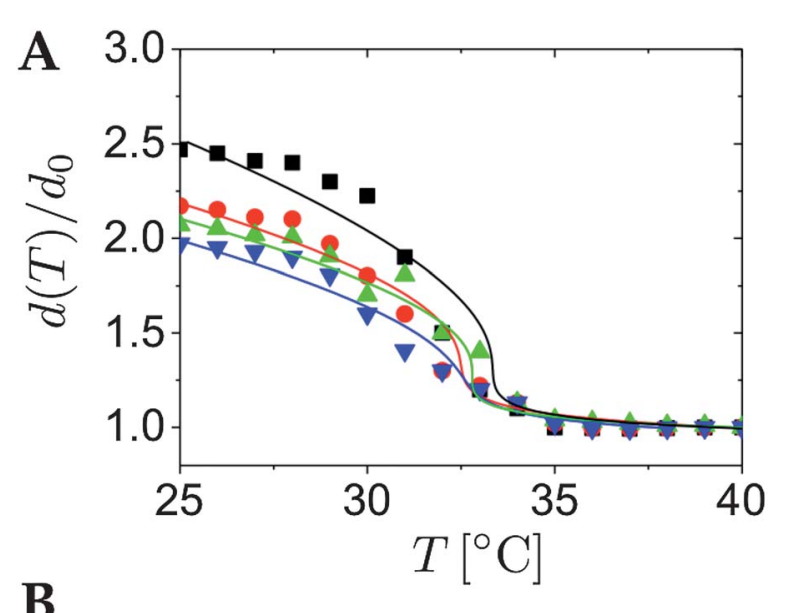

B

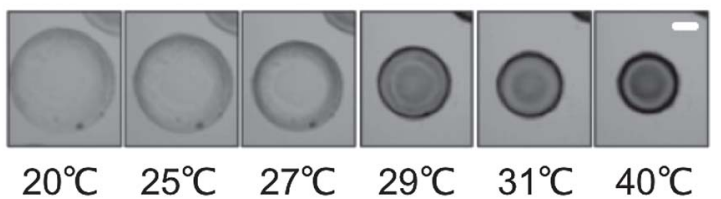

Fig. 1 Deswelling transition of pNIPAM microgels as a function of temperature. (A) Particle diameter scaled to its value in the fully collapsed state $d / d_{0}$ as a function of temperature $T$ for different monomer crosslinker ratios (squares: 1/36, circles: 1/14, up triangles: 1/10, down triangles: 1/7). Solid lines through each measurement denote a prediction of the Flory-Rehner theory. (B) Microscopy images of single pNIPAM microgels at different temperatures. The scale bar corresponds to $50 \mu \mathrm{m}$. experimental setup consisted of a tapered glass capillary with a taper angle $\alpha$ with the capillary inlet connected to a flexible tube, as shown schematically in Fig. 2(A). At the start of an experiment a dilute suspension of particles was injected into the capillary by applying a pressure $p$ to the flexible tube. To do so we use the hydrostatic pressure in the tube, which we controlled by varying the filling height.

As the diameter of the capillary at the tip is smaller than the diameter of the particles, the first particle that approaches the tip will block the further flow of the fluid in the capillary. As a result, the entire pressure difference $p$ between the inlet and the outlet of the capillary now falls off across this particle. As the applied pressure is increased, the resulting external forces acting on the particle cause it to move closer to the tip of the capillary and the particle deforms both in the shape as well as in the volume, as shown in Fig. 2(B). In equilibrium, when the particle no longer moves or deforms, the externally applied stress must balance the internal elastic stress of the particle.

The particle deformation as a function of $p$ is characterized with an optical microscope, enabling us to directly quantify the elastic properties of the particle. In the experiments on our samples we increased the applied pressure in steps from $p=$ $100 \mathrm{~Pa}$ to $p=9 \mathrm{kPa}$ and monitored the resulting elastic
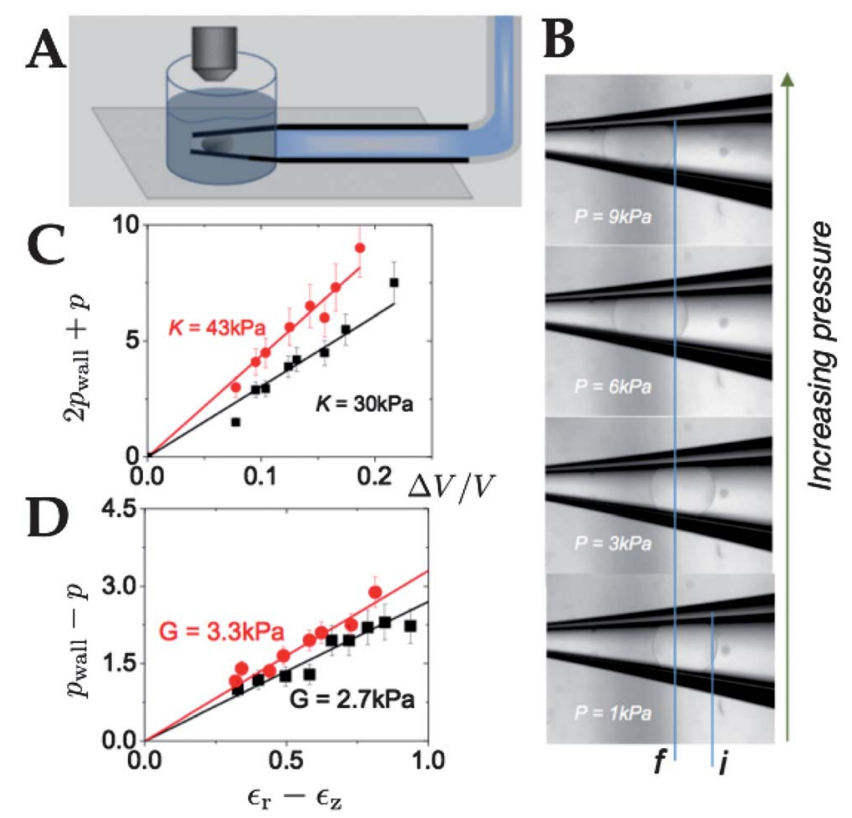

Fig. 2 Capillary micromechanics measurement. (A) Schematic of the experimental setup. A tapered glass capillary is connected at the inlet to a flexible tube and at the outlet into a reservoir. A suspension of particles is injected by applying a pressure $p$ to the inlet; flow stops when a single particle becomes trapped near the tip of the capillary. (B) Series of images of a single microgel particle at one fixed temperature $(T=23 \mathrm{~K}$ ). As the applied pressure $p$ was increased (from bottom to top), the particle moved closer to the tip of the capillary and changed both its shape as well as its volume in the process. The initial and final rear positions of the particle are indicated for clarity as $i$ and $f$, respectively. (C) Plot of the characteristic stress for compression, $\left(2 p_{\text {wall }}+p\right) / 3$, as a function of $\Delta V / V$; the slope of this curve corresponds to the compressive elastic modulus $K$. (D) Characteristic stress for shear deformation, $\left(p_{\text {wall }}-p\right) / 2$, as a function of the characteristic strain $\varepsilon_{\mathrm{r}}-\varepsilon_{z i}$ the slope of this curve is the shear elastic modulus $G$. 
deformation of a particle, as seen in Fig. 2(B). It can clearly be seen in the images that our PNIPAM particles changed not just their shape, but also their volume in the process. We characterized the volume from the images by taking into account that the shape of the particles is rotationally symmetric around the central axis of the glass capillary. To characterize the changing shape of the particles we focussed on the contact surface between the particle and the glass wall, which has the shape of a tapered circular band. In order to quantify both the changes in shape as well as volume of the particles, we extracted the positions of 6 characteristic points on the acquired images: the foremost and rearmost points of the particles along the central axis of the capillary, as well as the four points at the edge of the contact band between the particle and the glass wall of the capillary.

We denote the length of this band along the flow direction as $L_{\text {band }}$ and the average radius of this band as $R_{\text {band }}$, which we use as a measure of the longitudinal strain deformation $\varepsilon_{z}$ and the radial strain deformation, $\varepsilon_{\mathrm{r}}$, respectively. The particle volume was calculated from the positions of the 6 characteristic points by assuming that the particle is rotationally symmetric along the central axis of the capillary and consists of 3 sub-volumes: a frustum of a cone with circular cross-section at the center, and two sphere caps at the front and the rear of the particle. ${ }^{11,29}$ In this simple description of the particle deformation, the parameters $V, R_{\text {band }}$ and $L_{\text {band }}$ are sufficient to describe the shape and volume change of the particle in order to analyze its elastic properties.

In equilibrium, the externally applied stress on the particle is balanced by the internal elastic stresses. This balance allows us to quantify the elastic properties of the material in terms of the compressive elastic modulus $K$ and the shear elastic modulus $G$. The external forces exerted on the particle by the walls of the capillary are quantified by a wall pressure $p_{\text {wall }}$; this is the average force per unit area acting on the contact surface between the particle and the glass capillary. From the balance of the externally applied forces acting on the particle, the wall pressure is derived as

$$
p_{\text {wall }}=\frac{p}{\gamma_{\mathrm{f}} \cos \alpha+\sin \alpha} \frac{R_{\mathrm{wall}}}{2 L_{\mathrm{wall}}},
$$

where $\alpha$ is the taper angle of the capillary. Static friction at the walls exerts a net friction force $F_{\mathrm{f}} \approx \gamma_{\mathrm{f}} p_{\text {wall }} 2 \pi R_{\text {band }} L_{\text {band }} \cos \alpha$ acting on the particle with a friction coefficient $\gamma_{\mathrm{f}}$. As friction between pNIPAM microgels and glass surfaces is in general very low, in the range of $\gamma_{\mathrm{f}}=10^{-4}$ to $10^{-3},{ }^{11,36}$ we have neglected the effects of friction by setting $\gamma_{\mathrm{f}}=0$ in eqn (1). The wall pressure can then be written as

$$
p_{\text {wall }}=\frac{p}{\sin \alpha} \frac{R_{\text {wall }}}{2 L_{\text {wall }}} .
$$

Finally, we assumed that the stress is isotropic inside the particle and identified the externally applied stress in the flow direction with the applied pressure difference $p$ and in the radial direction with the wall pressure $p_{\text {wall }}$. For an isotropic material these elastic stresses can be written as a function of $K$ and $G$, as well as the three-dimensional strain deformations as

$$
\sigma_{\mathrm{r}}=K\left(2 \varepsilon_{\mathrm{r}}+\varepsilon_{z}\right)+\frac{2}{3} G\left(\varepsilon_{\mathrm{r}}-\varepsilon_{z}\right)
$$

and

$$
\sigma_{z}=K\left(2 \varepsilon_{\mathrm{r}}+\varepsilon_{z}\right)-\frac{4}{3} G\left(\varepsilon_{\mathrm{r}}-\varepsilon_{z}\right) .
$$

Balancing these externally applied stresses with the internal elastic stress yields the elastic compressive modulus

$$
K=\frac{1}{3} \frac{2 p_{\text {wall }}+p}{2 \varepsilon_{\mathrm{r}}+\varepsilon_{z}},
$$

and the shear elastic modulus

$$
G=\frac{1}{2} \frac{p_{\text {wall }}-p}{\varepsilon_{\mathrm{r}}-\varepsilon_{z}} .
$$

These expressions are physically intuitive if we consider that $\left(2 p_{\text {wall }}+p\right)$ and $\frac{1}{2}\left(p_{\text {wall }}-p\right)$ can be seen as characteristic stresses for a volumetric compression and a shear deformation, respectively. Similarly, the volumetric strain $\Delta V / V \approx\left(2 \varepsilon_{\mathrm{r}}+\varepsilon_{z}\right)$ characterizes a pure volume deformation, while $\left(\varepsilon_{\mathrm{r}}-\varepsilon_{z}\right)$ can be seen as a characteristic strain for a shear deformation, characterizing a change in shape. ${ }^{\mathbf{1 1 2 9}}$ Our analysis was thus performed by plotting the characteristic stress for compression and for shear as a function of the corresponding characteristic strain, as shown in Fig. 2(C) and (D), respectively. The red circles show results for the particles with an initial cross-linker-to-monomer molar ratio of $1 / 14$, while the black squares show results for the softest particles studied, for which during synthesis we used a cross-linker-to-monomer molar ratio of $1 / 36$.

\section{Results and discussion}

\subsection{Particle size as a function of temperature}

The most prominent temperature-dependent property of pNIPAM particles is the collapse transition that occurs as the temperature is increased across the lower critical solution temperature. Our particles are synthesized via drop-based microfluidics, yielding typical sizes on the order of tens of micrometers. We were thus able to observe the deswelling transition of the particles directly in an optical microscope, equipped with a temperature-controlled sample chamber (Linkam Scientific Instruments, model TP-93/LNP). A typical example of this temperature-dependent deswelling is shown for a single microgel particle in the series of images in Fig. 1(B). The particle size decreased with temperature and finally, well above the LCST, at $T>38{ }^{\circ} \mathrm{C}$ the particle size reached a fully collapsed state.

In order to rationalize the size dependence as a function of temperature and cross-linker concentration, we compared our results to Flory-Rehner theory, which has been extensively used to describe the behavior of macroscopic hydrogels and has also recently been applied to microgels and minigels. ${ }^{7,9,37}$

According to Flory-Rehner theory the swelling equilibrium at each temperature corresponds to the conditions where the total osmotic pressure acting on the gel, $\Pi_{\text {total }}$, is zero. In this context, the osmotic pressure arises from two main 
contributions. The first important contribution is the osmotic pressure due to mixing, expressed as

$$
\Pi_{\mathrm{mix}}=\frac{k_{\mathrm{B}} T}{a^{3}}\left[\phi+\ln (1-\phi)+\chi \phi^{2}\right],
$$

with $k_{\mathrm{B}}$ the Boltzmann constant, $\chi$ the Flory parameter that accounts for the solubility of the polymer in the solvent, $a$ the monomer size, and $\phi$ the polymer volume fraction within the particle. The second contribution to consider is the elastic contribution to the osmotic pressure, expressed as

$$
\Pi_{\text {elastic }}=\frac{k_{\mathrm{B}} T \phi_{0}}{N_{\mathrm{gel}} a^{3}}\left[\frac{\phi}{2 \phi_{0}}-\left(\frac{\phi}{\phi_{0}}\right)^{\frac{1}{3}}\right],
$$

where $\phi_{0}$ is the polymer volume fraction within a particle in its collapsed state, and $N_{\text {gel }}$ is the average number of monomers between cross-links.

In equilibrium, the total osmotic pressure difference has to vanish, and therefore, in the absence of additional contributions to the pressure, these two contributions have to balance each other, $\Pi_{\text {tot }}=\Pi_{\text {mix }}+\Pi_{\text {elastic }}=0$. Hence,

$$
\frac{\phi_{0}}{N_{\text {gel }}}\left(\frac{\phi}{2 \phi_{0}}-\left(\frac{\phi}{\phi_{0}}\right)^{\frac{1}{3}}\right)=\left(\phi+\ln (1-\phi)+\chi \phi^{2}\right) \text {. }
$$

The Flory solvency parameter $\chi$ describes the interactions of the polymer with the solvent and depends in principle on the temperature and the polymer concentration. ${ }^{34}$ The $\phi$-dependence of $\chi$ is expressed by expanding $\chi$ in a power series of $\phi$,

$$
\chi=\chi_{1}+\chi_{2} \phi+\chi_{3} \phi^{2}
$$

where we presume the temperature-dependent part of the $\chi$ parameter to be represented by $\chi_{1}$, which can be expressed as

$$
\chi_{1}=\frac{1}{2}-A\left(1-\frac{\theta}{T}\right)
$$

with $\theta$ the so-called theta temperature, defined as the temperature where the excess chemical potential of mixing between the polymer and the solvent is zero and the polymers behave like ideal chains. For our PNIPAM system, the theta temperature is above the critical temperature of the volume phase transition. The parameter $A$ is also a phenomenological parameter that we determined by curve fitting to our experimental data. We presumed the parameters $\chi_{2}$ and $\chi_{3}$ to be temperature independent for reasons of simplicity.

Finally, we assumed that the particles exhibit an isotropic swelling, implying that the particle size cubed is inversely proportional to the volume fraction, according to $\left(\frac{d}{d_{0}}\right)^{3}=\frac{\phi_{0}}{\phi}$. By introducing our expression for the $\chi$ parameter in eqn (9), one obtains an explicit equation of state; for brevity we do not display it here but instead refer to eqn (3) of a recent paper by Sierra-Martin et $a l^{27}$ This equation of state relates the temperature to the polymer volume fraction in the microgel. In the following, we compare the predictions that follow from this equation of state to our experimental data.

As it stands, we have six free parameters, being $\phi_{0}, A, \theta, \chi_{2}, \chi_{3}$ and $N_{\text {gel }}$ which obviously is rather excessive. It turns out that we actually "only" need four, that is, we can set $\chi_{2}=\chi_{3}=0$ to get reasonable fits for our deswelling curves. (Below, we shall be studying the influence of $\chi_{2}$ and $\chi_{3}$ in predicting the outcome of our mechanical experiments.) Unfortunately, we are able to obtain fits with similar quality (similar standard deviation) for quite different sets of values of these four parameters, $\phi_{0}, A, \theta$ and $N_{\text {gel }}$. In order to make headway and make the curve fitting more meaningful, we limited the parameters $N_{\text {gel }}$ and $\phi_{0}$ to physically meaningful values, in line with previously reported values, ${ }^{7,27,31}$ making our procedure in a way a two- rather than a four-parameter fit.

For instance, the average number of monomers between cross-links can be estimated from the synthesis by considering that each cross-linker molecule connects two chains and hence has 4 connections leading to other cross-links. So, the number concentration of the chains connecting two cross-linkers should be around twice that of the number of cross-linkers themselves. So, for example, for 1/36 molar fraction of crosslinker to monomer, we expect $N_{\text {gel }} \approx 72$. This is indeed very close to the value of 62 that we obtain from a fit to the data, using $\phi_{0}=0.7$, as shown in Table 1 , entry (i). We obtained an even better agreement for the 1/14 molar ratio sample, where we expected $N_{\text {gel }} \approx 28$ and from our fit we obtained a value of 28 , as shown in Table 1 , entry (ii). Thus, choosing an appropriate value of $\phi_{0}$ and limiting $N_{\text {gel }}$ to a physically meaningful range, we obtained a reasonable fit to our data, as shown in Fig. 1(A), where at the same time the obtained fitting parameters are in agreement with what is expected from the microgel synthesis and from previous experimental results. ${ }^{7,27,31}$

\subsection{Particle modulus as a function of temperature}

Along with the significant deswelling of the particles, we also expect dramatic changes in their mechanical properties upon increasing the temperature across the LCST. In order to study these changes in single-particle mechanics, we measured the elastic properties of the particles across the volume phase transition by using the recently developed capillary micromechanics technique, ${ }^{\mathbf{1 1}, 29}$ as described in the Experimental section. By plotting the characteristic stress for compression and for shear as a function of the characteristic strains, as shown in Fig. 2(C) and (D), the compressive and shear moduli were determined, respectively, as the slope of these plots. At

Table 1 Parameters from fits to the temperature-dependent particle diameter $d(T)$, according to eqn (9), where $\phi_{0}$ and $N_{\text {gel }}$ were fixed and the other parameters used as free parameters. Fits (i), (ii-a), (iii) and (iv) are shown in Fig. 1(A) as solid lines. Fits (ii-a), (ii-b) and (ii-c) are shown in Fig. 4(A). The same parameters are also used in Fig. 4(B)-(D), where the Flory-Rehner predictions for $\sigma(T), K(T)$, and $G(T)$, respectively, are compared to the experimental data

\begin{tabular}{llllllll}
\hline Fit & $\phi_{0}$ & $d_{0}$ & $N_{\text {gel }}$ & $A$ & $\Theta$ & $\chi_{2}$ & $\chi_{3}$ \\
\hline (i) & 0.7 & 1 & 62 & -7 & 318 & 0 & 0 \\
(ii-a) & 0.69 & 0.97 & 28 & -3.73 & 318 & 0 & 0 \\
(ii-b) & 0.67 & 0.97 & 22 & -4.45 & 320 & -0.4 & 0 \\
(ii-c) & 0.62 & 0.97 & 22 & -5.15 & 321 & -0.76 & 0.42 \\
(iii) & 0.69 & 1 & 17.2 & -5.8 & 318 & 0 & 0 \\
(iv) & 0.67 & 0.98 & 13 & -5.2 & 318 & 0 & 0
\end{tabular}


room temperature, a linear fit to our data yields the shear elastic moduli of $G=2.7 \mathrm{kPa}$ and $G=3.3 \mathrm{kPa}$ for $1 / 36$ and $1 / 14$ crosslinker-to-monomer molar fractions. The data show linear behavior up to the highest deformations probed, indicating a linear elastic response. In analogy, for the compressive moduli we obtain $K=30 \mathrm{kPa}$ and $K=43 \mathrm{kPa}$, respectively for the two different cross-linker-to-monomer ratios. For larger cross-linking densities we expect higher moduli, which is indeed what we found in our experiments, even though the observed differences are relatively small, close to the standard deviation of approximately $10 \%$ observed in our measurements (see also in the $\mathrm{ESI}_{\dagger}^{\dagger}$ ).

By performing similar measurements at different temperatures we thus quantified the full elastic behavior of the particles as a function of temperature. Results are shown in Fig. 3(A) and (B), where we plot the compressive elastic modulus $K$ (solid squares, left axis) and the shear elastic modulus $G$ (open circles, left axis) as a function of temperature for particles synthesized with a cross-linker-to-monomer molar ratio of $1 / 36$ and $1 / 14$, respectively. For both types of particles we observed qualitatively the same temperature dependence of their elastic properties. The shear modulus $G$ of the particles increased

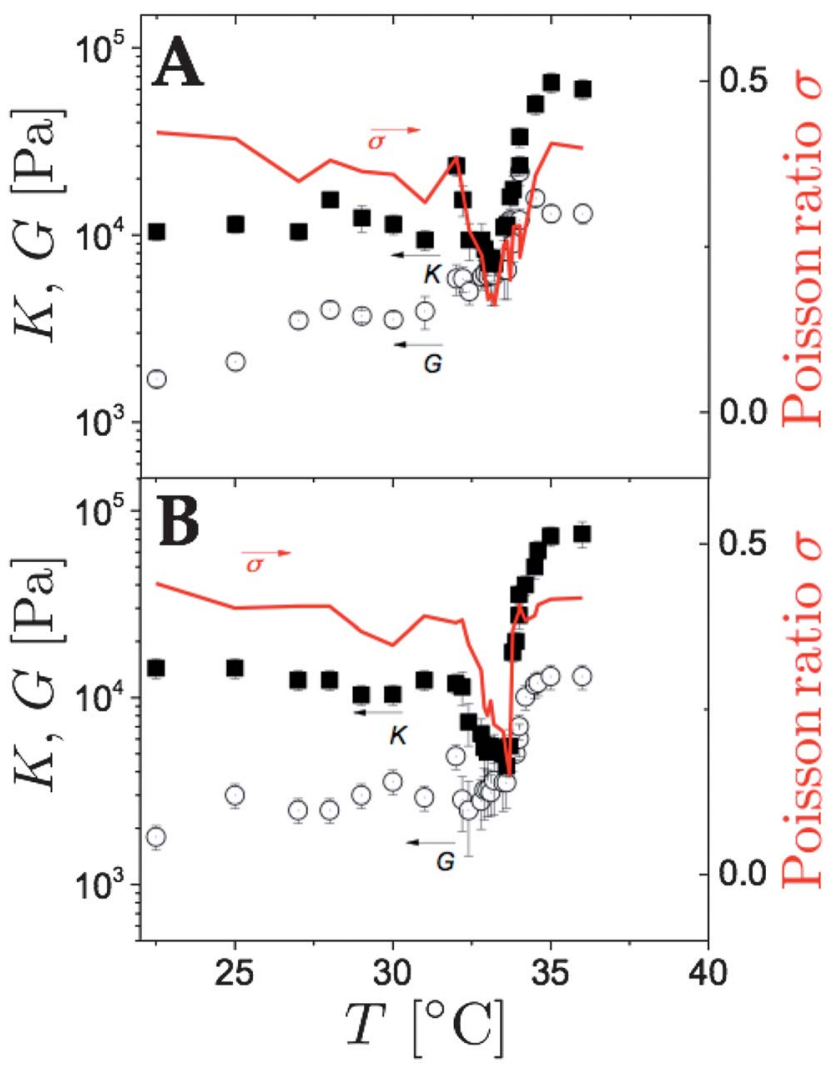

Fig. 3 Elastic properties as a function of temperature for particles prepared with initial cross-linker-to-monomer ratios of 1/36 (A) and 1/14 (B). In both cases, the compressive modulus $K$ (solid squares, left axis) exhibited a pronounced decrease close to the LCST, while the shear modulus $G$ (open circles, left axis) increased continuously. As a result, near the LCST the Poisson ratio $\sigma$ of the material (solid line, right axis) dropped significantly at the LCST, reaching minimum values of $\sigma=$ 0.15 (A) and $\sigma=0.17$ (B), respectively. continuously with increasing temperature, with the steepest increase taking place around the LCST of the material at around $33{ }^{\circ} \mathrm{C}$. In contrast, the compressive elastic modulus $K$ of the particles exhibited a pronounced dip around the LCST, first decreasing from around $10 \mathrm{kPa}$ at room temperature to around $6 \mathrm{kPa}(\mathrm{A})$ or $4 \mathrm{kPa}(\mathrm{B})$. Finally, above the LCST, $K$ increased again to a value of around $100 \mathrm{kPa}$ in the collapsed state of the particles.

This qualitatively different temperature-dependence of $K$ and $G$ implies that the material does not behave like a conventional polymer gel in the absence of an observable collapse transition, where the ratio between the two moduli would not change dramatically. Intuitively, we can relate these differences to the volume phase transition that occurs at the LCST; as the particles deswell, there is no physical reason for a drop in the shear modulus $G$, which is expected to increase as a result of the increase in the polymer concentration within a particle and the increase of attractive interactions between the polymer chains. Conversely, around the LCST, a small change in temperature leads to a dramatic change in the particle volume. In the absence of a large increase of the heat capacity of the material in this temperature range, the dramatic deswelling of the particles thus indicates that the energy required to change the particle volume should be significantly lowered. As a result, we would intuitively expect a drop of the compressive elastic modulus around the LCST of the material.

The dramatic changes in mechanical behavior of these microgel particles can be further illustrated by considering the Poisson ratio $\sigma$ of the material as a function of temperature. The Poisson ratio, given as,

$$
\sigma=\frac{3 K-2 G}{2(3 K+2 G)}
$$

describes the deformation of a material under a uniaxial load; if a material is compressed in one direction with a strain $\varepsilon_{z}$, then the deformation in the perpendicular directions is given as $\varepsilon_{x, y}=\sigma \varepsilon_{z}$. For incompressible materials, $\sigma=1 / 2$; this applies to most common liquids and solid materials.

Here, as often observed for polymer gels, ${ }^{32}$ we observe a significant deviation from incompressible material behavior, as shown in Fig. 3(A) and (B), where the Poisson ratio is plotted as a solid red line (right axis). At the lowest temperatures studied, we found a Poisson ratio of $\sigma \approx 0.4$, still relatively close to incompressible behavior. However, as the temperature was increased, we observed a significant drop of $\sigma$, reaching values close to zero in the vicinity of the LCST; the Poisson ratio reached a minimum of $\sigma \approx 0.17$ and $\sigma \approx 0.15$ for cross-linkerto-monomer ratios of $1 / 36$ and $1 / 14$, respectively. Above the LCST, the Poisson ratio returned to a more conventional value of $\sigma \approx 0.4$.

A Poisson ratio of zero is unusual for common materials, but is theoretically expected for polymer gels under poor solvent conditions. ${ }^{32}$ Such values close to zero have indeed been previously observed for polymer gels ${ }^{32}$ and for macroscopic pNIPAM hydrogels, where even negative values have been reported. ${ }^{30,31,38,39}$ In these latter studies, comparisons to FloryRehner theory have indicated that the theory can at least 
qualitatively account for the observed mechanical behavior. Fits of Flory-Rehner theory to the mechanical behavior were performed, yielding fair agreement with experimentally observed mechanical properties. However, in principle, the deswelling behavior of the material, according to eqn (9), should be sufficient to obtain the relevant parameters for the Flory-Rehner theory. These parameters could then be used to predict the mechanical behavior, based on the observed temperaturedependent deswelling behavior.

In the following, to test whether Flory-Rehner theory can indeed account for the behavior observed in our experiments, we use the model parameters obtained from fits to the temperature-dependent particles size in order to predict the mechanical behavior. According to Flory-Rehner theory the compressive elastic modulus $K$ is given by

$$
K=\frac{k_{\mathrm{B}} T}{a^{3}}\left[\frac{\phi^{2}}{1-\phi}-2 \chi \phi^{2}+\frac{\phi_{0}}{N_{\mathrm{gel}}}\left(\frac{\phi}{\phi_{0}}-\left(\frac{\phi}{2 \phi_{0}}\right)^{1 / 3}\right)\right],
$$

where $k_{\mathrm{B}} T$ is the thermal energy, $a$ is the molecular size of a monomer, and the $\phi$-dependence of the Flory parameter is described by eqn (10). In addition, the shear elastic modulus $G$ is given by

$$
G=\frac{\phi_{0} k_{\mathrm{B}} T}{2 N_{\mathrm{gel}} a^{3}}\left(\frac{\phi}{\phi_{0}}\right)^{1 / 3} .
$$

To test the validity of the theory, we performed several fits to the temperature-dependent particle size, shown in Fig. 4(A), where the experimental data are shown as full circles. In describing the Flory solvency parameter according to eqn (10) we have previously set the higher order parameters $\chi_{2}$ and $\chi_{3}$ to zero, which yielded reasonably good curve fits to our deswelling data, shown by the red dotted line in Fig. 4(A). This curve fitting should in principle predict the mechanical behavior as well. However, as shown in Fig. 4(B)-(D), where we plot $K, G$ and $\sigma$ as a function of temperature, the prediction is at best qualitative: the theoretical dip is much sharper and deeper than observed in our experiments. It stands to reason that including the higher order parameters $\chi_{2}$ and $\chi_{3}$ could produce a better agreement.

In order to test the influence of including these higher order parameters, we performed two additional fits, where $\chi_{2}$ and $\chi_{3}$, describing the $\phi$-dependence of $\chi$, are included as additional fit parameters. The resulting fits are shown in Fig. 4. For the green dashed lines $\chi_{2}$ was used as a fit parameter, and for the blue solid lines both $\chi_{2}$ and $\chi_{3}$ were used.

The obtained parameters are listed in Table 1, as fits number (iii-v). The quality of these three fits to the deswelling data does not change significantly, as shown in Fig. 4(A), where the three curves largely overlap. Nevertheless, if we use these same parameters for the prediction of the storage and loss moduli, as given by eqn (13) and (14), significant differences are observed. While all three curves are able to predict the qualitative

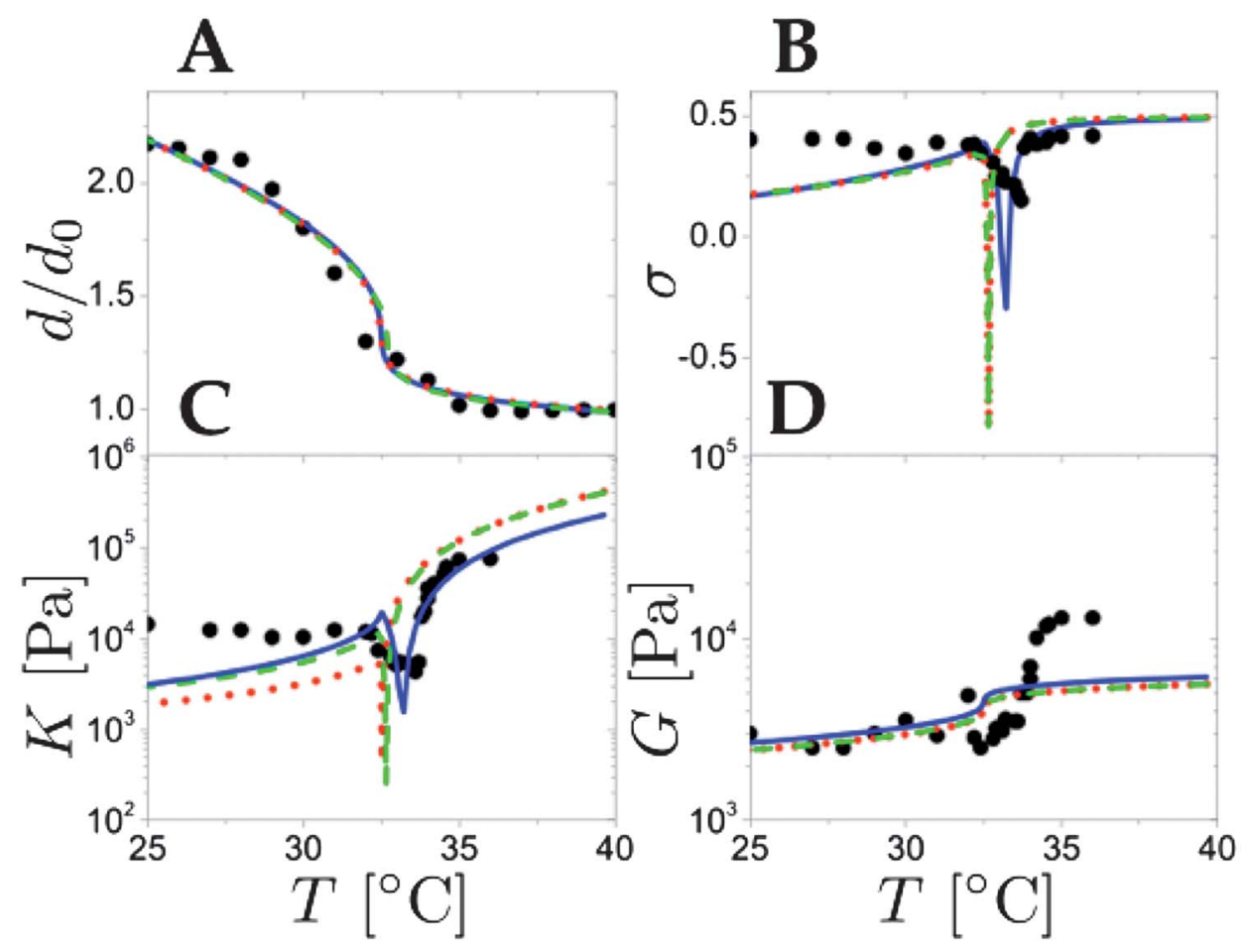

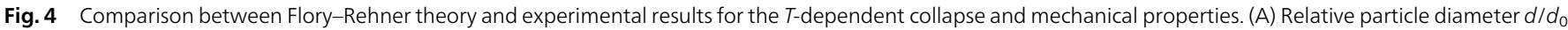

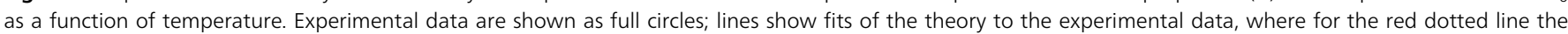

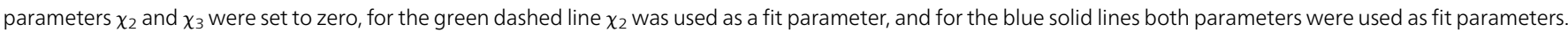

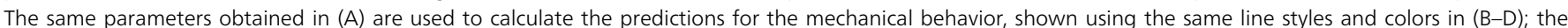

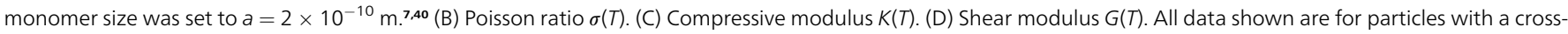
linker to monomer molar ratio of $1 / 14$. 
behavior observed in the experiment, the contribution of higher orders in the $\phi$-dependence of $\chi$ appears to lead to a closer agreement with the mechanical experiments. The fit with the highest order in the $\phi$-dependence of $\chi$ more closely matches the experimental data than do the lower order fits, as shown in Fig. 4(C); the minimum in $K(T)$ is less pronounced and broader, in closer agreement with the experimental data. For the elastic shear modulus $G(T)$, all three curves lie on top of each other, as here the dependence on $\chi$ is not important, as readily evident from eqn (14).

However, while the theory does predict a sharp increase in $G$ around the LCST, the experimentally observed increase is much more pronounced. Eqn (14) predicts an increase of $G$ inversely proportional to the particle diameter, or proportional to the cube root of the polymer concentration within the particle, $G \propto \phi^{1 / 3}$. As pointed out by Hashmi and Dufresne, ${ }^{7}$ a scaling of $G \propto \phi$, as predicted by conventional rubber elasticity theory, would be in much closer agreement with experimental data for both pNIPAM hydrogels and for the microgels studied here, where $G \propto \phi$ would imply approximately a ten-fold increase in $G$ over the temperature range indicated, which would be in close agreement with our experimental observations.

As a result, while Flory-Rehner theory can account for the general behavior observed in our experiments, it does not quantitatively predict the full behavior. Most striking is its failure to predict the scaling of the elastic shear modulus across the transition; it severely underestimates the $\phi$-dependence of the elastic shear modulus, as shown in Fig. 4(D).

\section{Conclusions}

Our experiments for the first time characterize the full elastic response of pNIPAM microgels as the temperature is varied across the LCST of the material. As found in earlier studies for bulk hydrogels we found a non-monotonic temperature dependence for the compressive modulus, which exhibits a pronounced dip in the vicinity of the LCST. Also similar to bulk hydrogels, the shear modulus does not exhibit such a dip, instead increasing continuously as the temperature is increased. As a result of the qualitatively different temperaturedependences of $K$ and $G$, the Poisson ratio of the particles shows a pronounced minimum close to the LCST, reaching values close to zero.

Physically, this behavior is the result of the free energy required to change the particle volume, proportional to $K$, dropping to very low values, while the free energy required to change the particle shape, proportional to $G$, steadily increases. As a result, for macroscopic hydrogels, even negative Poisson ratios have been observed close to the LCST, which indicates that it costs less free energy to change the volume than it does to change the shape of a piece of material. For similar synthesis conditions as those used in the studies on macroscopic PNIPAM hydrogels by Hirotsu, ${ }^{31}$ we did not quite observe a negative Poisson ratio, but merely a dip to values close to zero. The depth of the observed minimum in $\sigma$ follows the general trend observed also in the macroscopic case; as the cross-linking density decreases, the minimum value of $\sigma$ decreases and eventually becomes negative at low enough cross-linking densities. While this trend is confirmed in our experiments, for the lowest cross-linking densities we could achieve, the observed Poisson ratio remained positive. Our attempts to further decrease the cross-linking density of our particles did not lead to the formation of structurally intact microgels. However, it is possible that negative Poisson ratios could also be achieved in microgels if the cross-linking density could successfully be further decreased, while keeping the integrity of the microgel particles intact.

While the general trends observed for the elastic behavior of macroscopic gels and our microgel experiments are similar, the dip observed in our experiments for the Poisson ratio and the compressive modulus as a function of temperature are much broader than those seen in macroscopic gels. This could be a result of the finite size of the particles, leading to a qualitatively different behavior in the case of microgels than is observed for hydrogels of macroscopic dimensions. Such a trend is also in agreement with earlier experiments on microgel particles in which only the compressive modulus ${ }^{27}$ or the Young's modulus $E$ of single microgel particles ${ }^{7}$ was measured as a function of temperature; in both cases, a much broader dip in $K$ or $E$, respectively, is observed than for the case of macroscopic gels. The physical reason for this apparent finite-size effect is still unclear. Interestingly, recent experiments on the mechanics of single pNIPAM polymer chains also appear to follow the same trend, with single polymer chains representing the smallest possible system size. ${ }^{\mathbf{4 1}}$ In these experiments a dip in the single chain spring constant was observed as a function of temperature; this dip is much broader than those observed in macroscopic gels and even broader than for our microgel particles. This indicates that in order to quantitatively predict the mechanical behavior observed in both microgels and macroscopic hydrogels, a new theoretical approach is needed, which must specifically account for the observed finite size effects.

\section{References}

1 L. A. Lyon and A. Fernandez-Nieves, Annu. Rev. Phys. Chem., 2012, 63, 25-43.

2 A. Fernandez-Barbero, I. J. Suarez, B. Sierra-Martin, A. Fernandez-Nieves, F. Javier de las Nieves, M. Marquez, J. Rubio-Retama and E. Lopez-Cabarcos, Adv. Colloid Interface Sci., 2009, 147-148, 88-108.

3 J. M. Griffin, I. Robb and A. Bismarck, J. Appl. Polym. Sci., 2007, 104, 1912-1919.

4 R. Pelton, Adv. Colloid Interface Sci., 2000, 85, 1-33.

5 G. Romeo, A. Fernandez-Nieves, H. M. Wyss, D. Acierno and D. A. Weitz, Adv. Mater., 2010, 22, 3441-3445.

6 J.-J. Lietor-Santos, B. Sierra-Martin, R. Vavrin, Z. Hu, U. Gasser and A. Fernandez-Nieves, Macromolecules, 2009, 42, 6225-6230.

7 S. M. Hashmi and E. R. Dufresne, Soft Matter, 2009, 5, 36823688.

8 J. Mattsson, H. M. Wyss, A. Fernandez-Nieves, K. Miyazaki, Z. B. Hu, D. R. Reichman and D. A. Weitz, Nature, 2009, 462, 83-86. 
9 B. Sierra-Martin, J. A. Frederick, Y. Laporte, G. Markou, J. J. Lietor-Santos and A. Fernandez-Nieves, Colloid Polym. Sci., 2011, 289, 721-728.

10 J. Wiedemair, M. J. Serpe, J. Kim, J. F. Masson, L. A. Lyon, B. Mizaikoff and C. Kranz, Langmuir, 2007, 23, 130-137.

11 H. M. Wyss, T. Franke, E. Mele and D. A. Weitz, Soft Matter, 2010, 6, 4550-4555.

12 S. Adams, W. J. Frith and J. R. Stokes, J. Rheol., 2004, 48, 1195.

13 T. Tanaka and D. J. Fillmore, J. Chem. Phys., 1979, 70, 12141218.

14 I. J. Suarez, A. Fernandez-Nieves and M. Marquez, J. Phys. Chem. B, 2006, 110, 25729-25733.

15 J. Wang, D. Gan, L. A. Lyon and M. A. El-Sayed, J. Am. Chem. Soc., 2001, 123, 11284-11289.

16 M. Goldberg, R. Langer and X. Jia, J. Biomater. Sci., Polym. Ed., 2007, 18, 241-268.

17 J. B. Thorne, G. J. Vine and M. J. Snowden, Colloid Polym. Sci., 2011, 289, 625-646.

18 J. K. Oh, R. Drumright, D. J. Siegwart and K. Matyjaszewski, Prog. Polym. Sci., 2008, 33, 448-477.

19 Y. Qiu and K. Park, Adv. Drug Delivery Rev., 2012, 53, 321339.

20 Microgel Suspensions: Fundamentals and Applications, ed. Alberto Fernandez-Nieves, Hans M. Wyss, Johan Mattson and David A. Weitz, John Wiley \& Sons, 2011, p. 500.

21 J. Stokes and W. Frith, Soft Matter, 2008, 4, 1133-1140.

22 J. J. Litor-Santos, B. Sierra-Martn and A. Fernndez-Nieves, Phys. Rev. E: Stat., Nonlinear, Soft Matter Phys., 2011, 84, 060402.

23 E. H. Purnomo, D. van den Ende, J. Mellema and F. Mugele, Europhys. Lett., 2006, 76, 74-80.

24 S. Schmidt, M. Zeiser, T. Hellweg, C. Duschl, A. Fery and H. Mhwald, Adv. Funct. Mater., 2010, 20, 3235-3243.
25 P. A. Fernandes, S. Schmidt, M. Zeiser, A. Fery and T. Hellweg, Soft Matter, 2010, 6, 3455-3458.

26 M. Stieger, W. Richtering, J. S. Pedersen and P. Lindner, J. Chem. Phys., 2004, 120, 6197-6206.

27 B. Sierra-Martin, Y. Laporte, A. B. South, L. A. Lyon and A. Fernndez-Nieves, Phys. Rev. E: Stat., Nonlinear, Soft Matter Phys., 2011, 84, 011406.

28 H. M. Wyss, J. M. Henderson, F. J. Byfield, L. A. Bruggeman, Y. X. Ding, C. F. Huang, J. H. Suh, T. Franke, E. Mele, M. R. Pollak, J. H. Miner, P. A. Janmey, D. A. Weitz and R. T. Miller, Am. J. Physiol.: Cell Physiol., 2011, 300, C397-C405. 29 M. Y. Guo and H. M. Wyss, Macromol. Mater. Eng., 2011, 296, 223-229.

30 S. Hirotsu, Macromolecules, 1990, 23, 903-905.

31 S. Hirotsu, J. Chem. Phys., 1991, 94, 3949.

32 E. Geissler and A. M. Hecht, Macromolecules, 1980, 13, 12761280.

33 P. J. Flory and J. Rehner Jr, J. Chem. Phys., 1943, 11, 521.

34 P. J. Flory, J. Chem. Phys., 1950, 18, 108.

35 R. K. Shah, J. W. Kim, J. J. Agresti, D. A. Weitz and L. Y. Chu, Soft Matter, 2008, 4, 2303-2309.

36 T. Tominaga, N. Takedomi, H. Biederman, H. Furukawa, Y. Osada and J. P. Gong, Soft Matter, 2008, 4, 1033-1040.

37 A. Fernandez-Nieves and A. Fernndez-Barbero, J. Chem. Phys., 2003, 119, 10383.

38 S. Hirotsu, Phase Transitions: A Multinational Journal, 1994, 47, 183-240.

39 C. Li, Z. Hu and Y. Li, Phys. Rev. E: Stat. Phys., Plasmas, Fluids, Relat. Interdiscip. Top., 1993, 48, 603-606.

40 A. Fernandez-Barbero, A. Fernandez-Nieves, I. Grillo and E. Lopez-Cabarcos, Phys. Rev. E: Stat., Nonlinear, Soft Matter Phys., 2002, 66, 051803.

41 S. Cui, X. Pang, S. Zhang, Y. Yu, H. Ma and X. Zhang, Langmuir, 2012, 28, 5151-5157. 